\title{
Leiomyoma of the uterine round ligament: A case report
}

\author{
ALIKI TYMPA $^{1}$, CHARALAMPOS GRIGORIADIS ${ }^{2}$, EMMANOUIL TERZAKIS ${ }^{2}$, \\ CHRISTINA GOUDELI $^{2}$ and AIKATERINI MELEMENI ${ }^{1}$ \\ ${ }^{1}$ First Department of Anaesthesiology, Aretaieion Hospital, University of Athens, Medical School, Athens 11528;
${ }^{2}$ Department of Obstetrics and Gynaecology, Leto Maternity Hospital, Athens 11524, Greece
}

Received January 28, 2021; Accepted July 6, 2021

DOI: $10.3892 / \mathrm{etm} .2021 .10720$

\begin{abstract}
Leiomyomas are common benign tumours that can arise in any anatomical structure containing smooth muscle. Their localization in the uterine round ligament is rare, although leiomyomas are the most frequent tumour of this structure. Leiomyomas present as inguinal, labial or intra-abdominal masses, and are often misdiagnosed as hernias or enlarged lymph nodes. The aim of the present study was to describe a rare case of a large intra-abdominal mesenchymal neoplasm arising from the right round ligament of the uterus. A 51-year-old asymptomatic female patient (gravida 3, para 3) presented herself for a routine gynaecological examination. A transvaginal ultrasound examination revealed a solid heterogeneous mass with a maximum diameter of $9 \mathrm{~cm}$ localized at the right parametrial space. Further preoperative evaluation using magnetic resonance imaging revealed that the mass contained solid and cystic components, which was suggestive of a mesenchymal neoplasm with possible involvement of the right ovary. Complete excision of the tumour and total abdominal hysterectomy with bilateral salpingo-oophorectomy was performed via laparotomy under general anaesthesia. The intraoperative findings, frozen section biopsies and final histological examination of the tumour established the diagnosis of an intra-abdominal myoma of the right uterine round ligament. The majority of abdominal round ligament myomas are initially asymptomatic. The role of synchronous imaging examinations, such as ultrasonography and magnetic resonance imaging, in the diagnosis of these lesions is crucial.
\end{abstract}

\section{Introduction}

The uterine round ligament is a round, rope-like band of fibromuscular connective tissue (1). One side of the ligament

Correspondence to: Dr Charalampos Grigoriadis, Department of Obstetrics and Gynaecology, Leto Maternity Hospital, 7-13 Mouson Street, Athens 11524, Greece

E-mail: xarisgrigoriadis@yahoo.gr

Key words: leiomyoma, uterus, round ligament, ultrasonography is attached to the superior and lateral aspect of the uterus at the anatomical location of the uterine cornu. Arising from the cornu of the uterus, the round ligament is covered by peritoneum over a length of $10-12 \mathrm{~cm}$, crosses the pelvis via the deep inguinal ring, then traverses the inguinal canal and finally enters the labia majora, where it terminates with its fibres blending into the mons pubis (1).

Leiomyomas are benign tumours that may arise in any anatomical structure containing smooth muscle (2). However, in contrast to typical leiomyomas, which are a relatively common gynaecological problem, leiomyomas of the uterine round ligament are rare (2). Tumours of the round ligament are exceedingly rare, with roughly 300 cases previously published in the literature (3). Although rare, leiomyomas are the most frequent tumour of the uterine round ligament (3). Other tumours encountered at the uterine round ligament according to histological type include adenomyomas, mesothelial cysts, endometriotic cysts, leiomyosarcomas and other, even rarer entities (4).

Leiomyomas of the uterine round ligament may appear in multiple different anatomical locations at any point along the length of this structure, and are commonly classified as intra- or extra-abdominal (inguinal or labial). Intra-abdominal leiomyomas of the uterine round ligament typically remain asymptomatic. On pelvic and ultrasound examination, the differential diagnosis of leiomyomas from pedunculated subserosal myomas or solid ovarian neoplasms is difficult (4). Even following computed tomography and magnetic resonance imaging examinations, the lack of specific findings, as leiomyomas appear as encapsulated heterogeneous tumours, hinders final diagnosis prior to surgical intervention and histological examination (5). On the other hand, extra-abdominal leiomyomas are frequently detected by the patient as a mass lesion with synchronous symptoms, including pain, and differential diagnosis from hernias or enlarged lymph nodes is necessary $(4,6)$.

In the present study, a rare case of a large leiomyoma of the right uterine round ligament detected during routine gynaecological examination is described, in line with the Surgical CAse REport (SCARE) criteria (7). The aim was to underline the role of routine gynaecological examination and the importance of complete preoperative diagnostic evaluation in cases of large intra-abdominal lesions to plan the appropriate surgical approach and ensure optimal management. 


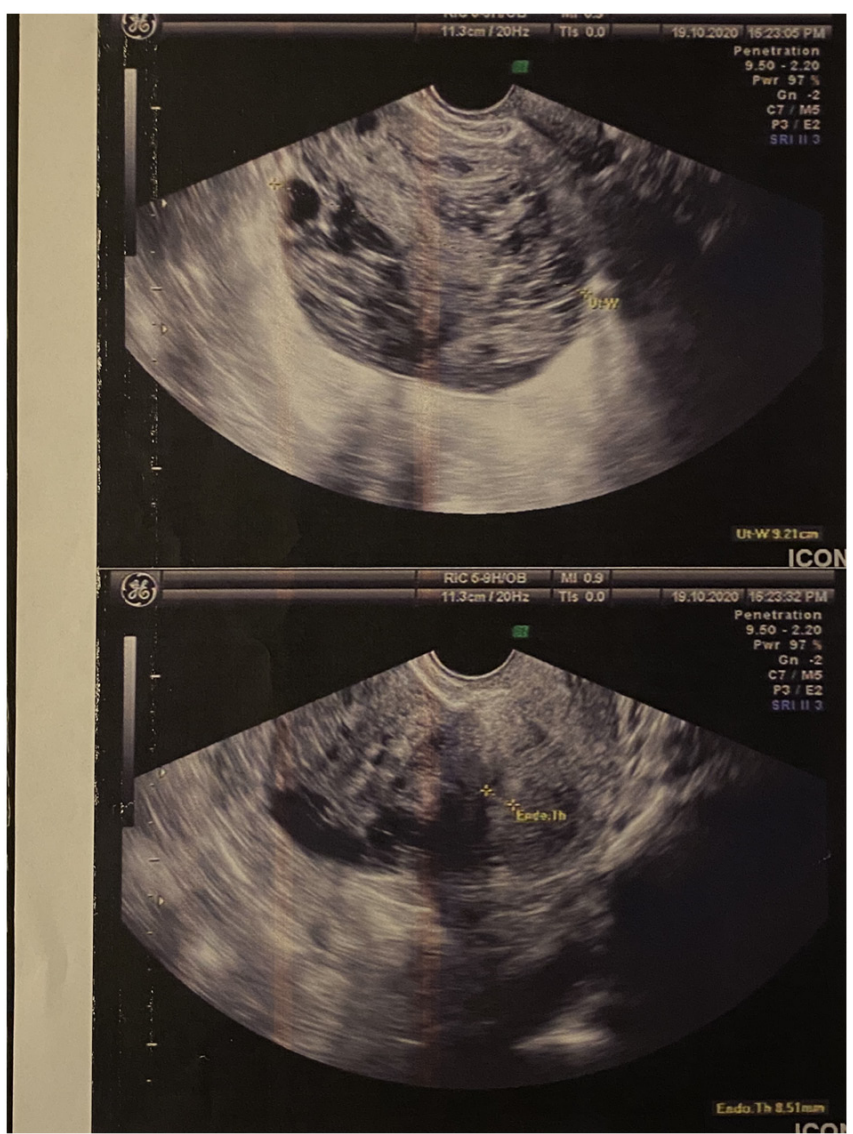

Figure 1. Transvaginal ultrasonographic appearance of a large solid tumour of the right parametrium attached to the uterus (maximum tumour diameter, 9.2-cm; thickness of the endometrium, $8.5 \mathrm{~mm})$.

\section{Case report}

A 51-year-old asymptomatic female patient (gravida 3, para 3) presented herself on October 2020 for a routine gynaecological examination. The patient had obstetrical history of three full-term vaginal deliveries and no previous surgical interventions, while her medical history was unremarkable.

A transvaginal ultrasound examination revealed a solid heterogeneous mass localized at the right parametrial space with a maximum diameter of $9 \mathrm{~cm}$, which was suggestive of a large pelvic lesion with possible involvement of the right ovary (Fig. 1). Routine blood investigations and tumour marker levels were within the normal range. Further preoperative evaluation using magnetic resonance imaging revealed that the mass (maximum diameter, 9-cm) contained solid as well as cystic components, which was suggestive of a mesenchymal neoplasm, possibly originating from the right ovary. There were no enlarged pelvic lymph nodes or ascites.

After obtaining informed consent from the patient, a laparotomy was performed at Leto Maternity Hospital (Athens, Greece) on November 2020, under general anaesthesia via a midline vertical subumbilical incision. A large mobile solid mass arising from the right uterine round ligament was detected (Fig. 2). Complete excision of the lesion was performed (Fig. 3). The frozen section biopsy revealed a mesenchymal neoplasm without mitotic activity or signs

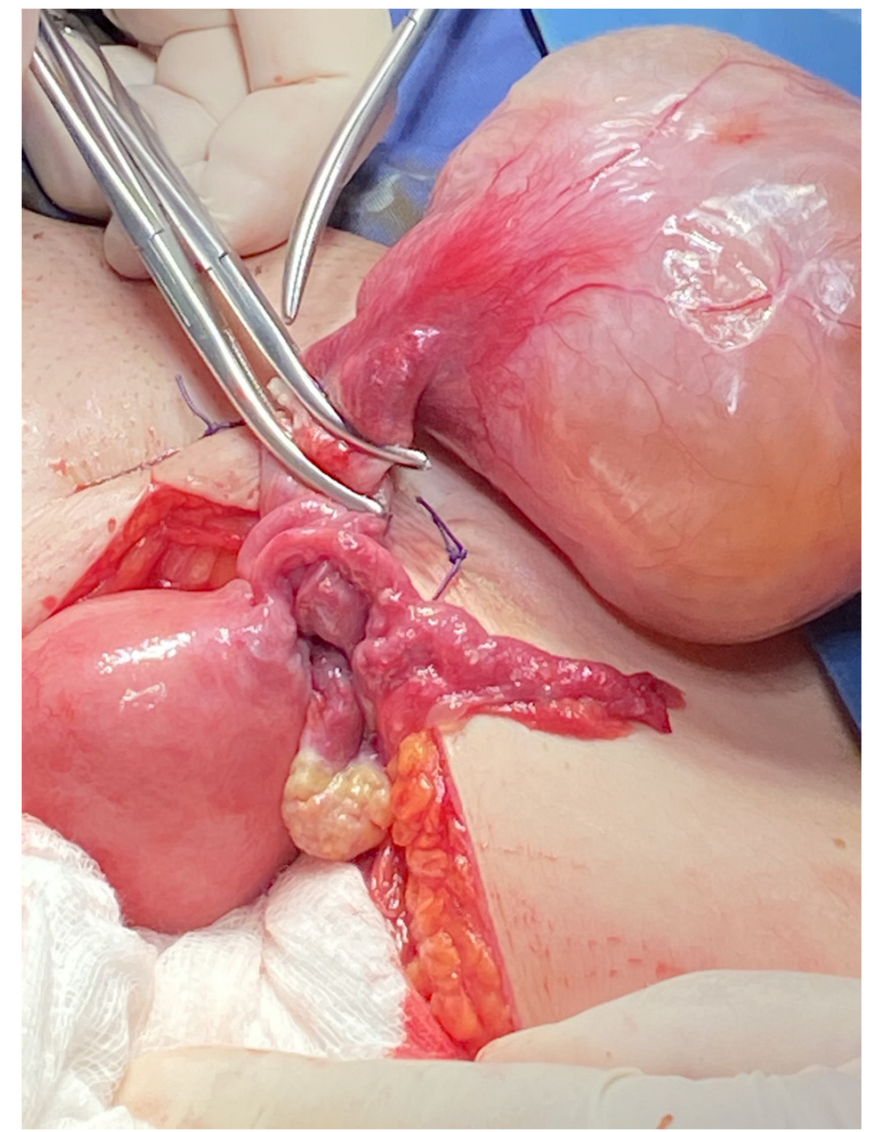

Figure 2. Surgical excision of the tumour following dissection from the right uterine round ligament.

of necrosis, with morphological characteristics suggestive of a benign leiomyoma of the uterine round ligament. Total abdominal hysterectomy and bilateral salpingo-oophorectomy were subsequently performed.

Histological examination revealed a benign leiomyoma with a maximum diameter of $10.5 \mathrm{~cm}$, with signs of fibrosis, hyalinosis and oedema. On immunohistological examination, the neoplastic cells were positive for desmin and smooth muscle actin, as expected. High density of mononuclear inflammatory cells, mainly small lymphocytes with perivascular location, were detected. Among them, rare eosinophil leukocytes and mastocytes were recognised (data not shown). There were no malignant findings from the uterus, cervix oradnexae. The postoperative period was uneventful and the patient was discharged on postoperative day 2 without complications. No recurrence was identified until her last follow-up visit on June 2021.

\section{Discussion}

Leiomyomas of the uterine round ligament are rare, and may present with multiple clinical manifestations according to the location along the anatomical course of the ligament. Symptoms associated with leiomyomas largely depend upon location, size and rate of growth (4). In the vast majority of cases, intra-abdominal leiomyomas of the round ligament remain asymptomatic, and diagnosis is often made incidentally during routine ultrasound examination or surgical 


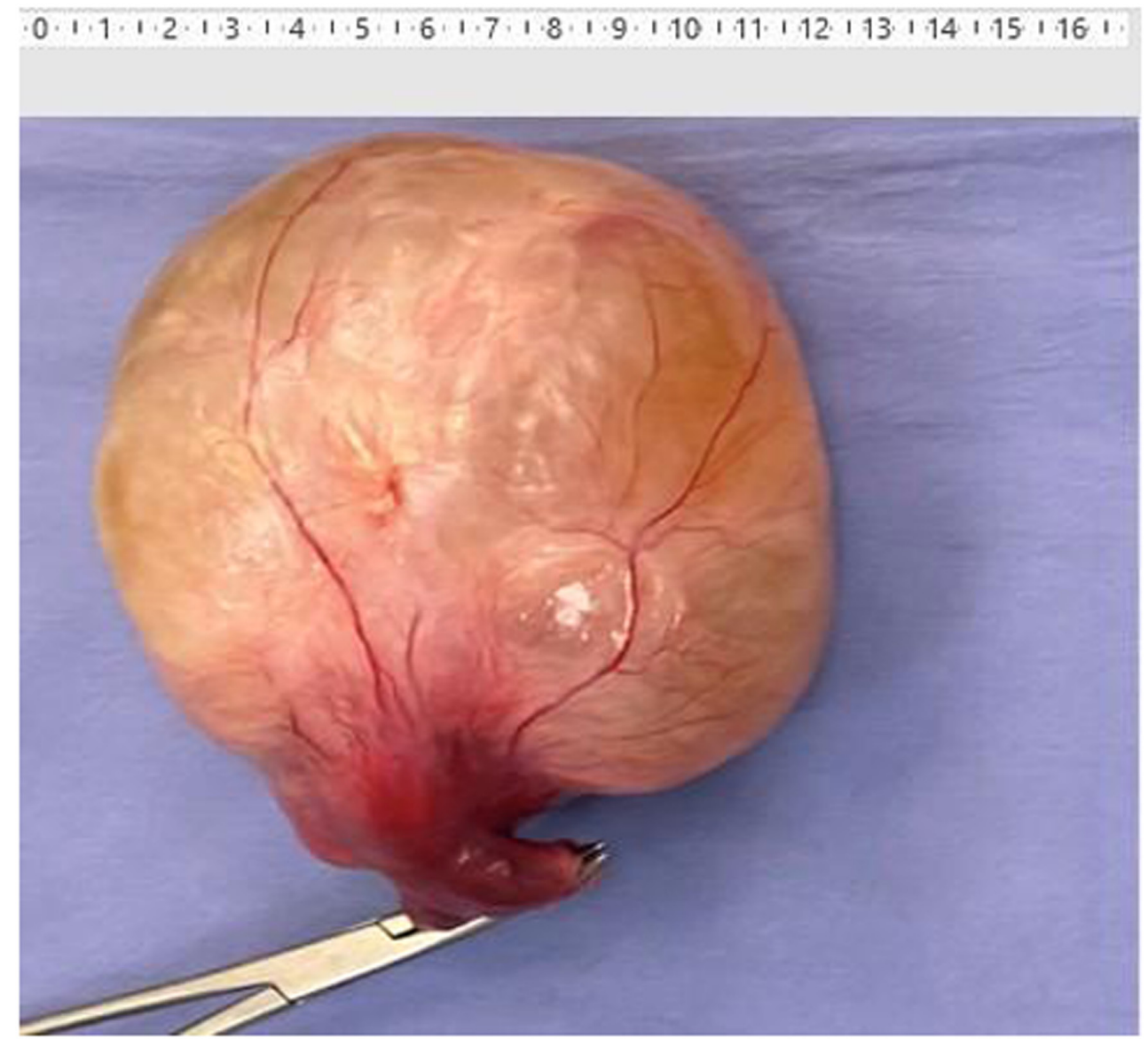

Figure 3. Surgical specimen of neoplasm arising from the right round ligament of the uterus.

intervention for other reasons $(3,4)$. These literature data agree with the history of the patient in the present case, as she was asymptomatic and the tumour was detected on routine gynaecological examination. The patient's last transvaginal ultrasound gynaecological examination had been performed 4 years earlier, without signs of pathology at the right parametrium, which suggested that the solid heterogeneous mass identified in the present study was a pelvic tumour with a high rate of growth. Surgical intervention with oncological parameters was recommended as a safe therapeutic strategy for a tumour with a potentially aggressive biological behaviour. This was the reason for which laparotomy was suggested instead of laparoscopic excision of the tumour in order to avoid morcelation. In addition, the tumour was sent for frozen section biopsy so as to have a first histological view and decide if further pelvic/paraaortic lymphadenectomy was necessary or not.

Preoperative investigation via computed tomography or magnetic resonance imaging often fails to distinguish the origin of solid pelvic tumours. Leiomyomas appear as encapsulated heterogeneous tumours on spontaneous contrast-enhanced computed tomography and post-gadolinium contrast magnetic resonance imaging (5). The definitive differential diagnosis from subserosal myomas or solid ovarian neoplasms often requires surgical intervention. In the present case, magnetic resonance imaging was unable to identify the origin of the pelvic mass, and final diagnosis was made intraoperatively.
Leiomyomas of the uterine round ligament are primarily solitary and unilateral, although they have also been reported to be multiple and bilateral (4). A number of studies have reported that leiomyomas appear more frequently on the right compared with the left uterine round ligament $(4,8,9)$. Although the aforementioned studies support this predilection for the right round ligament, which was also the case in our patient, there appears to be no medical explanation for this observation.

Surgical intervention is currently considered as the most appropriate therapeutic strategy in cases of large pelvic tumours with uncertain differential diagnosis. Complete excision of the lesions is necessary in cases of uterine leiomyomas, without the requirement for hysterectomy in women of reproductive age (2-4). The patient presented herein was a perimenopausal 51-year-old woman, and the total abdominal hysterectomy with bilateral salpingo-oophorectomy that followed the excision of the leiomyoma of the right uterine round ligament was performed as a preventive measure.

Routine gynaecological examination is necessary to prevent women from experiencing life-threatening pathological conditions that affect the reproductive system. The vast majority of patients may be asymptomatic, as abdominal tumours arising from the ovaries or the uterus may not give rise to symptoms, even when they reach a large size. Surgical intervention remains the optimal therapeutic strategy $(3,4)$, following appropriate preoperative evaluation, as a means of 
definitive diagnosis, as well as curative treatment in cases of large intra-abdominal tumours with a challenging differential diagnosis.

\section{Acknowledgements}

Not applicable.

\section{Funding}

No funding was received.

\section{Availability of data and materials}

The datasets used and/or analyzed during the current study are available from the corresponding author on reasonable request.

\section{Authors' contributions}

AT was involved in the study conception and design, data collection and analysis and the writing of the manuscript. CG participated in the writing of the manuscript, data collection and data analysis. ET was involved in the study design and data analysis. CG was involved in data analysis. AM was involved in study conception and design. All authors have read and approved the final manuscript. CG, ET and CG confirm the authenticity of the raw data.

\section{Ethics approval and consent to participate}

The present study was approved by the Ethics Committee of Leto Maternity Hospital (approval no. 38/2021).

\section{Patient consent for publication}

The patient provided written informed consent for the publication of the case details and any associated images.

\section{Competing interests}

The authors declare that they have no competing interests.

\section{References}

1. Chaudhry SR and Chaudhry K: Anatomy, abdomen and pelvis, uterus round ligament. In: StatPearls [Internet]. StatPearls Publishing, Treasure Island, FL, 2021. https://www.ncbi.nlm.nih. gov/books/NBK499970/. Accessed July 26, 2021.

2. Kirkham JC, Nero CJ, Tambouret RH and Yoon SS: Leiomyoma and leiomyosarcoma arising from the round ligament of the uterus. J Am Coll Surg 207: 452, 2008.

3. Klingbeil KD, Polcari AM, Azab B and Franceschi D: Large, extra-abdominal leiomyoma of the round ligament with carneous degeneration. BMJ Case Rep 2017: bcr2017222454, 2017.

4. Breen JL and Neubecker RD: Tumors of the round ligament: A review of the literature and report of 25 cases. Obstet Gynecol 19: 771-780, 1962.

5. Michel P and Viola D: Abdomino-pelvic leiomyoma of the round ligament: Contribution of computed tomography and magnetic resonance imaging. J Gynecol Obstet Biol Reprod (Paris) 32: 571-574, 2003 (In French).

6. Christodoulou IM, Angelopoulos A, Siaperas P, Ioannidis A, Skarpas A, Tellos A, Velimezis G and Karanikas I: Leiomyoma of the round ligament of the uterus mimicking inguinal hernia. Case Rep Surg 2018: 6702494, 2018.

7. Agha RA, Borrelli MR, Farwana R, Koshy K, Fowler A and Orgill DP; SCARE Group: The SCARE 2018 statement: Updating consensus Surgical CAse REport (SCARE) guidelines. Int J Surg 60: 132-136, 2018.

8. Emanuel R: Veber tumoren des ligamentum rotundum uteri. Ztschr f Geburtsh u Gynak Stuttgart 49: 383, 1903.

9. Taussig FG: Sarcoma of the round ligament of the uterus. Surg Gynec Obst 19: 218, 1914.
This work is licensed under a Creative Commons
Attribution-NonCommercial-NoDerivatives 4.0 International (CC BY-NC-ND 4.0) License. 